\title{
Comparação da função cognitiva de idosas institucionalizadas e das participantes de centro de convivência
}

\author{
Vanessa Lôbo de Carvalho*, Daniela Vilella de Carvalho Medeiros*, \\ Jillaner Camila Alexandre dos Santos Silva*", Samara Gomes Barbosa*", Laís Leandro de Souza**
}

\section{Resumo}

O objetivo deste artigo é comparar a função cognitiva entre idosas institucionalizadas e aquelas que participam de centro de convivência. Trata-se de um estudo quantitativo, observacional e transversal, em que se aplicou o Mini Exame do Estado Mental (MEM), para detectar alterações cognitivas em quarenta idosas, sendo que vinte delas eram residentes em Instituições de Longa Permanência para Idosos (ILPIs), e vinte participavam de centro de convivência. A amostra caracterizou-se pela média de 74,05 de idade, com desvio padrão de 4,99. Para comparar os domínios, foi utilizado o teste não paramétrico de Mann-Whitney, com nível de significância de $5 \%$. Os domínios de orientação no tempo e no espaço apresentaram uma diferença estatisticamente significativa $(p<0,05)$, entre as idosas participantes do centro de convivência e as idosas institucionalizadas. As participantes do centro de convi- vência apresentaram maiores escores nos domínios anteriormente citados. A conclusão do estudo foi de que as idosas, residentes nas ILPIs, apresentaram um escore menor, nos domínios espaciais e temporais, quando comparadas às participantes de um centro de convivência.

Palavras-chave: Envelhecimento. Cognição. Participação Social. Instituição de Longa Permanência para Idosos.

\section{Introdução}

A população idosa, definida como o conjunto de indivíduos com mais de sessenta anos (em países subdesenvolvidos e emergentes), e a que está acima de 65 anos (nos países desenvolvidos), tem aumentado consideravelmente (JACINTO, 2008).

* Fisioterapeuta. Mestra em Ensino na Saúde no contexto do SUS (UFAL). Universidade Estácio Alagoas, Maceio (AL). Endereço: Rua Professor Lourenço Peixoto, 31 - Jutiúca - Maceió (AL) CEP 57035-640. E-mail: carvalhovanessa@hotmail.com Fisioterapeuta. Mestra em Ensino na Saúde no contexto do SUS (UFAL). Universidade Estácio Alagoas, Maceio (AL). Endereço: Rua Professor Lourenço Peixoto, 31 - Jutiúca - Maceió (AL) CEP 57035-640. E-mail: carvalhovanessa@hotmail.com.

** Graduadas em Fisioterapia pela Universidade Estácio de Alagoas, Maceió (AL). E-mails: danimedeirosv@hotmail.com; jillaner_camila@hotmail.com; laisleandro90@hotmail.com; samaragomes.b@ hotmail.com.

$\rightarrow$ http://dx.doi.org/10.5335/rbceh.2014.3409

Recebido em: 15/08/2013. Aprovado em: 30/07/2014 
O envelhecimento é conceituado como um processo dinâmico e progressivo no qual há modificações na homeostase, nos aspectos morfológicos, funcionais, bioquímicos e psicológicos, o que ocasiona progressiva perda da capacidade de adaptação do indivíduo ao meio ambiente, gerando maior vulnerabilidade e maior incidência de processos patológicos (SILVA et al., 2007).

Há dois conceitos que caracterizam o envelhecimento: a senescência e a senilidade. A senescência é um processo natural do ciclo vital, no qual ocorre uma deterioração física e mental. A senilidade refere-se a um processo patológico, em que há um declínio físico mais acentuado e uma desorganização mental (SANTOS et al., 2010).

Conforme a segunda edição do guia de referência do idoso, elaborado pela Secretaria do Estado de Alagoas, existem, em Maceió, nove Instituições de Longa Permanência para Idosos (ILPIs) e 39 centros, grupos de convivência, clubes da melhor idade e associações para idosos.

As ILPIs são instituições governamentais ou não governamentais, de caráter residencial, destinadas a proporcionar domicílio coletivo a pessoas com idade igual ou superior a sessenta anos, com ou sem suporte familiar, em condição de liberdade, dignidade e cidadania (BORGES, 2008).

O Serviço Social do Comércio (SESC), de São Paulo, na década de 1970, deu início a um programa para a terceira idade, surgindo, assim, os grupos de convivência de idosos, que realizam diversas atividades, englobando aspectos culturais, sociais, educativos e de promoção da saú- de. Tais atividades vêm se multiplicando em associações comunitárias, centros de saúde, clubes, paróquias e instituições de ensino superior (CARVALHO FILHO; PAPALEO NETTO, 2000).

Com o aumento do número de idosos, existe uma maior preocupação com os problemas que atingem essa população, principalmente os de saúde e, em especial, os de ordem mental (NORDON et al., 2009).

Em relação ao envelhecimento cerebral, pode-se constatar que, quanto maior for a utilização de atividades intelectuais pelo idoso, mais tempo o seu encéfalo irá preservar suas conexões sinápticas, o que pode apresentar, como consequência, uma perda sintomática, tendo em vista sua capacidade plástica. Dessa forma, diversos processos ocorrem com o envelhecimento cerebral, dentre eles, atrofia cerebral, com dilatação de sulcos e ventrículos; presença de placas neuríticas; degeneração granulovacuolar, perda de neurônios, formação de corpos de lewy, a partir da alfa sinucleína, formação de placas beta-amilóides e emaranhados neurofibrilares. Tais lesões se iniciam a partir dos sessenta anos, nas regiões temporais mediais, e depois progridem para todo o neocórtex (NORDON et al., 2009; FERREIRA; RODRIGUES; PAIVA, 2008).

A cognição apresenta-se como uma função cortical, podendo ser dividida nas sub-funções: memória, atenção, orientação, raciocínio, função executiva, organização visuo-motora, solução de problemas e planejamento (FREITAS, 2011).

O que leva aos deficits cognitivos, comumente observados como naturais do envelhecimento, são algumas altera- 
ções características como: esquecimento de fatos recentes, alterações de atenção e dificuldades de cálculo (NORDON et al., 2009).

O declínio cognitivo encontra-se entre os diversos problemas que contribuem para a má qualidade de vida dos indivíduos. Assim, a procura de instrumentos de avaliação, principalmente de triagem cognitiva é fundamental para monitorar mudanças sutis na cognição, a fim de que doenças como a demência sejam identificadas em estágios precoces (MOTA et al., 2008).

Dentre os testes de triagem cognitiva, o Mini Exame do Estado Mental (MEEM) é o mais utilizado, para avaliação da função mental e cognitiva, tendo como objetivo rastrear alterações precoces, auxiliando as extensões das limitações, para o planejamento terapêutico (SANTOS et al., 2010).

De acordo com o Ministério da Saúde o Meem é um dos poucos testes de exame mental, indicado para ser utilizado nas Unidades Básicas de Saúde. Esse instrumento foi adaptado e validado ao Brasil, primeiramente, tem como vantagens a facilidade e a rapidez na sua aplicação cerca de dez minutos - (BERTOLUCCI et al.,1994; SANTOS et al., 2010).

O Meem possui trinta itens para avaliação de componentes dos processos cognitivos, segundo os subitens: (a) orientação do espaço, (b) orientação no tempo, (c) memória imediata, (d) atenção e cálculo, (e) memória de evocação, (f) linguagem e (g) habilidades visuo-espaciais (FERNANDES et al., 2009). Esse teste pode detectar alguns distúrbios do desempenho cognitivo em idosos, porém, é um instrumento clínico de avaliação, que exibe importante relação, com o nível de escolarização, e idade. Convém ressaltar que idosos com maior tempo de escolaridade preservam melhor sua capacidade intelectual e que estímulos a essa função são importantes promotores de melhoria, da memória (OLIVEIRA; BARROS; SOUZA, 2008).

Diversos autores brasileiros têm estabelecido pontos de corte diferenciados, de acordo com a idade (SANVITO, 2010). Brucki et al. (2003) realizam essa classificação, segundo o escore: Analfabetos-20 pontos; Escolaridade de 1 a 4 anos: 25 pontos; de 5 a 8 anos: 26,5 pontos; de 9 a 11 anos: 28 pontos; mais de 11 anos: 29 pontos (CHAVES, s.d.)

A população brasileira apresenta um grande número de idosos analfabetos, e com baixo nível educacional, em comparação aos demais países em desenvolvimento. $\mathrm{O}$ analfabetismo representa cerca de $30 \%$ dos idosos brasileiros (DINIZ; VOLPE; TAVARES, 2007).

Estudos mostram que exercícios físicos e mentais, moderados e contínuos, são eficazes na manutenção das capacidades cognitivas e são de grande importância, pois para Ericsson, Poston e Foreyt (1993), tais recursos são uma alternativa para a preservação da memória, do raciocínio e da percepção, dos indivíduos em processo de envelhecimento (FERREIRA; RODRIGUES; PAIVA, 2008).

Na busca de oferecer melhores condições para um envelhecimento saudável, na medida das possibilidades, este estudo objetivou comparar a função cognitiva em idosas institucionalizadas e as que 
participam de um centro de convivência, em uma capital do Brasil.

\section{Métodos}

Trata-se de um estudo quantitativo, observacional e transversal, realizado em duas Instituições de Longa Permanência para Idosos (ILPIs) e em um Centro de Referência de Assistência Social (CRAS), situados na cidade de Maceió, estado de Alagoas, no período de agosto, a outubro de 2012 .

O estudo foi composto por uma amostra de quarenta idosas, sendo vinte residentes em ILPIs, e vinte participantes de um grupo de convivência.

Os critérios de Inclusão no estudo foram: idosas com idades entre 65 e 85 anos, sem diagnóstico confirmado de doença mental; e a assinatura de termo de consentimento livre e esclarecido, pelas referidas idosas.

Os critérios de exclusão do estudo foram: declarar - pessoalmente ou por meio de informante - ser portador de deficiência visual e/ou auditivas graves, não corrigidas; estarem em estágios avançados de distúrbios cognitivos e/ ou doenças mentais, que pudessem impedir o entendimento e a execução dos procedimentos do teste; outro idioma materno, que não o português; dificuldade de movimentar as mãos, por doenças reumáticas ou neurológicas.

A amostra foi composta pela totalidade das idosas que preencheram o critério de inclusão. Não houve recusa dos sujeitos de pesquisa convidados.

Por se tratar de um teste de fácil e rápida execução, e de ampla utilização, o MEEM foi escolhido para detectar alterações cognitivas em pessoas, na prática clínica e em ensaios clínicos hospitalares e comunitários.

$\mathrm{Na}$ presente pesquisa, utilizou-se a versão brasileira do teste, sugerida no estudo de Brucki et al. (2003), adotada, também, por Fernandes et al. (2009) para avaliarem as funções cognitivas específicas e possui diversas questões, agrupadas em sete categorias: orientação para tempo (cinco pontos); registro de três palavras (três pontos); atenção e cálculo (cinco pontos); lembrança de três palavras (três pontos); linguagem (oito pontos); capacidade construtiva visual (um ponto). O referido teste consiste de comandos e perguntas, a serem dirigidas ao sujeito da pesquisa. O escore do Meem pode variar de um mínimo de zero, até um total de trinta pontos. A soma é realizada após o término do exame (KOPPER; TEXEIRA; DORNELES, 2009).

O processamento e a análise dos dados foram feitos por meio do software Statistical Package for the Social Sciences (SPSS) e, para realizar a comparação das porcentagens de acertos, entre as residentes das ILPIs e as participantes do grupo de convivência foi utilizado o teste não paramétrico de Mann-Whitney, adotando-se o nível de significância de $5 \%(0,05)$.

$\mathrm{O}$ instrumento foi aplicado por todas as pesquisadoras, após uniformização metodológica.

A pesquisa obteve a aprovação do Comitê de Ética em Pesquisa, da Faculdade Estácio de Alagoas, sob o parecer de n. 190612/025, conforme indicam as Resoluções n. 196/96 e 251/97, do Conselho Nacional de Saúde (CNS/MS). 


\section{Resultados}

No presente estudo, realizado com quarenta idosas, todas do sexo feminino, e faixa etária entre 65 e 85 anos, a amostra foi caracterizada com média de idade de 74,05 com desvio padrão de 4,99, sendo que, desse universo, vinte idosas residiam em ILPI's, e vinte participavam de um centro de convivência.

O Meem possui vários subitens, tais como: orientação temporal e espacial, leitura, atenção, cópia dos pentágonos e escrita. Todos os subitens sofrem influência da escolaridade. Para diminuir esse viés, são utilizados pontos de corte diferentes, de acordo com os anos de estudo. No entanto, existem divergências entre muitos autores, no que diz respeito aos pontos de corte a serem utilizados para esses escores (LENARDT et al., 2009). Utilizaram-se, no presente estudo, os pontos de corte propostos por Brucki et al. (2003), por serem considerados os mais adequados à amostra apresentada, tendo em vista a heterogeneidade do grau de escolaridade (Tabela 1).

Tabela 1 - Caracterização da amostra analisada no presente estudo

\begin{tabular}{l|c|c}
\hline Escolaridade & ILPIs & Centro de convivência \\
\hline analfabetas & $25 \%$ & $15 \%$ \\
$1-4$ anos & $25 \%$ & $20 \%$ \\
$5-8$ anos & $40 \%$ & $60 \%$ \\
$9-11$ anos & $5 \%$ & $5 \%$ \\
$>11$ anos & $5 \%$ & $0 \%$ \\
\hline
\end{tabular}

Fonte: primária.

Não foi possível realizar um estudo comparativo quanto à escolaridade, devido ao fato de a amostra apresentar diferenças numéricas que não permitiram a análise estatística. A pesquisa apresentou como fator limitante a amostra composta apenas pelo sexo feminino, pois há predominância desse sexo entre os participantes de centros de convivência e também entre os idosos institucionalizados.

Tabela 2 - Comparação dos subitens do MEEM

\begin{tabular}{l|c|c|c|c|c}
\hline \multirow{2}{*}{ Fator } & \multicolumn{2}{|c|}{ ILPls } & \multicolumn{2}{c|}{ Centro de convivência } & \multirow{2}{*}{ Valor de $p$} \\
\cline { 2 - 5 } & Média & Desv. Padrão & Média & Desv. Padrão & \\
\hline Orientação no tempo & 61,0 & 26,3 & 82,0 & 18,2 & $0,010^{*}$ \\
Orientação no espaço & 80,0 & 22,5 & 93,0 & 19,8 & $0,015^{*}$ \\
Memória imediata & 86,5 & 22,9 & 86,5 & 22,9 & 1,000 \\
Atenção e cálculo & 20,0 & 33,1 & 12,0 & 15,1 & 0,928 \\
Memória de evocação & 39,7 & 33,4 & 51,3 & 29,5 & 0,258 \\
Linguagem & 94,9 & 12,5 & 94,9 & 12,5 & 1,000 \\
Habilidade visuo-espacial & 61,5 & 31,2 & 76,5 & 21,2 & 0,116 \\
Resultado (Total) & 61,3 & 16,7 & 69,1 & 12,3 & 0,060 \\
\hline
\end{tabular}

Fonte: primária. 
Os resultados obtidos nesta pesquisa demonstraram que as participantes do centro de convivência apresentaram uma diferença estatisticamente significativa $(\mathrm{p}<0,05)$, em relação às idosas institucionalizadas, nos domínios de orientação no tempo e no espaço. Os demais domínios não apresentaram diferenças estatisticamente significativas (Tabela 2).

\section{Discussão}

O Meem não fornece diagnósticos demenciais, podendo ser utilizado para rastreamento de alterações cognitivas. No entanto, a piora na performance das atividades de vida diária, devido ao declínio cognitivo, é um marcador de quadros demenciais.

Lenardt et al. (2009) afirmam que a carência de outros estudos que utilizem o Meem, ligado à população de idosas que residem em ILPIs, dificulta a comparação dos resultados adquiridos no presente estudo, entretanto, confirmamos a importância da pesquisa.

No estudo de Bruno, Marques e Silva (2006), as idosas residentes nas ILPIs tiveram dificuldade no que se refere à avaliação da orientação temporal (data e dia da semana), e espacial (bairro e rua próxima). $\mathrm{O}$ autor acredita que a deficiência deve-se ao processamento da memória recente, acrescentando que é necessário o uso contínuo de lembretes, como calendários e anotações, com endereço e telefone, do local onde moram. No entanto, quando vivenciaram a pesquisa, essas pessoas demonstraram desinteresse em memorizar tais dados. No presente estudo foi observado que as participan- tes também apresentavam dificuldade nos subitens de orientação, no tempo e no espaço, mais significativamente, as residentes de ILPIs, em relação às participantes do centro de convivência.

Durante a presente pesquisa, foi observada a carência de objetos que estimulassem a memória (calendários, mapas, televisão, rádio, relógio, etc.), e também, que, nas instituições de longa permanência, existiam poucas atividades relacionadas à interação social, como as atividades em grupo, que auxiliam no desenvolvimento cognitivo e funcional.

O Cras é um órgão público municipal que tem como objetivo fornecer à população, em geral, serviços continuados de proteção básica de assistência social, principalmente àquelas pessoas que apresentem vulnerabilidade social. Todas as unidades possuem o Programa de Atenção Integral à Família, com o intuito de fortalecer os vínculos familiares e comunitários, através da atuação de equipes multiprofissionais (ALVARENGA et al., 2011). O grupo de convivência de idosos, avaliado no presente estudo, situa-se em um Cras, na cidade de Maceió-Alagoas.

No estudo de Santos; Foroni; e Chaves (2009), o escore médio, obtido pela amostra no Meem, foi de 24,09 pontos $(\mathrm{DP}=3,67)$. Os idosos que praticavam atividades de lazer obtiveram escores significativamente superiores, aos daqueles que não as praticavam, o que foi verificado pelo teste $t(p<0,05)$. Não houve diferença significativa $(p>0,05)$ de desempenho, no Meem, quando comparados os indivíduos que praticavam atividade física e aqueles que não 
praticavam. No que se refere ao lazer, no presente estudo não foi comprovada essa diferença, entretanto, quanto à prática de atividades físicas, o teste obteve resultados semelhantes aos obtidos no estudo de Santos; Foroni; e Chaves, já que, no escore total do Meem não houve diferença estatisticamente significativa, entre as idosas residentes em ILPIs (que não possuem atividade de lazer) e as participantes do grupo de convivência, que tem, como essência, atividades de lazer e físicas.

\section{Considerações finais}

Observa-se que houve uma diferença estatisticamente significativa entre as idosas residentes nas ILPIs, em comparação às idosas participantes de grupo de convivência, nos itens de orientação espacial e temporal, tendo como proposta de intervenção, a adoção de meios que possibilitem, às residentes em ILPIs, contatos com instrumentos que permitem o desenvolvimento e/ou a manutenção dos domínios espaciais e temporais. Tais instrumentos devem ser elaborados por uma equipe multiprofissional, com atuação interdisciplinar, para uma melhor adequação ao público-alvo e, se necessário, de forma individualizada.

\section{Comparison of cognitive function in elderly institutionalized and participants of living center}

\section{Abstract}

Objective: To compare cognitive function in institutionalized elderly participants and community center. Methods: This is a quantitative, observational and transversal. We used the mini-mental state examination for detecting cognitive changes in 40 elderly and 20 residents in homes for the aged and 20 participants in the community center. Results: The sample was characterized with a mean age of 74.05 with a standard deviation of 4.99. To compare the fields we used the nonparametric Mann-Whitney test with significance level of $5 \%$. The domains of orientation in time and space showed a statistically significant difference $(p<0.05)$ among the elderly participants of downtown living and institutionalized elderly. The participants of the community center had higher scores in the areas mentioned above. Conclusion: The elderly residents in home for the aged had a score lower in spatial and temporal domains when compared to participants in a community center.

Keywords: Aging. Cognition. Social Participation. Homes for the Aged.

\section{Referências}

ALAGOAS (Estado). Secretaria Estadual de Saúde. Guia de Referência do Idoso. 2. ed. 2010, 31p.

ALVAREGA, M. R. M. et al. Rede de suporte social do idoso atendido por equipes de Saúde da Família. Cien. Saude Colet., Rio de Janeiro, v. 16, n. 5, p. 2603-2611, maio 2011.

BERTOLUCCI, P. H. F. et al. O mini-exame do estado mental em uma população geral. Arq. Neuropsiquiatr., São Paulo, v. 52, n. 1, p. 1-7, mar. 1994. 
BORGES, P. L. C. Perfil dos idosos freqüentadores de grupos de convivência em Belo Horizonte, Minas Gerais, Brasil. Cad Saúde Publica, Rio de Janeiro, v. 24, n. 12, p. 27992808, dez. 2008.

BRUNO, C. T. S.; MARQUES, M. B.; SILVA, M. J. Avaliação cognitiva de idosos asilados utilizando o Miniexame do estado mental. Cad ESP., Fortaleza, v. 2, n. 1, p. 51-59, 2006.

BRUCKI, S. M. et al. Sugestões para o uso do mini-exame do estado mental no Brasil. Arq Neuropsiquiatr, São Paulo, v. 61, n. 3B, p. 777-781, set. 2003.

CHAVES, M. L. F. Testes de avaliação cognitiva: Mini-Exame do Estado Mental. Disponível em: <http://www.cadastro.abneuro. org/site/arquivos_cont/8.pdf $>$. Acesso em: 23 maio 2013.

DINIZ, B. S. O.; VOLPE, F. M.; TAVARES, A. R. Nível educacional e idade no desempenho no miniexame do estado mental em idosos residentes na comunidade. Rev. psiquiatr. clín., São Paulo, v. 34, n. 1, p. 13-17, 2007.

FERNANDES, R. C. L. et al. Avaliação da cognição de mulheres no climatério com o Mini-Exame do Estado Mental e o Teste de Memória da Lista de Palavras. Cad Saúde Publica, Rio de Janeiro, v. 25, n. 9, p. 18831893, set. 2009.

FERREIRA, T. A.; RODRIGUES, H. G.; PAIVA, L. R. Efeitos do envelhecimento sobre o encéfalo. RBCEH, Passo Fundo, v. 5, n. 2, p. 46-64, jul./dez. 2008.

CARVALHO FILHO, E. T.; PAPALEO NETTO, M. Geriatria: fundamentos, clínica e terapêutica. 2. ed. São Paulo: Atheneu, 2005, $788 \mathrm{p}$.

FREITAS, E.V. et al. Tratado de geriatria e gerontologia. Rio de Janeiro: Guanabara Koogan, 2011, 1750 p.

KOPPER, H.; TEIXEIRA, A. R.; DORNELES, S. Desempenho Cognitivo em um Grupo de Idosos: Influência de Audição, Idade, Sexo e Escolaridade. Arq. Int. Otorrinolaringol.,
São Paulo, v. 13, n. 1, p. 39-43, jan./jun. 2009.

JACINTO, A. F. Alterações cognitivas em pacientes idosos atendidos em ambulatórios geral de clínica médica. Tese (Neurologia) Faculdade de Medicina da Universidade de São Paulo, São Paulo, 2008.

LENARDT, M. H. et al. Desempenho de idosas institucionalizadas no miniexame do estado mental. Acta paul enferm., São Paulo, v. 22, n. 5, p. 638-644, 2009.

MOTA, M. M. P. E. et al. Triagem cognitiva: comparações entre o mini-mental e o teste de trilhas. Estud. psicol., Campinas-SP, v. 25, n. 3, p. 353-359, set. 2008.

NORDON, D. G. et al. Perda cognitiva em idosos. Rev. Fac. Ciênc. Méd. Sorocaba, Sorocaba, v. 11, n. 3, p. 5-8, set. 2009.

OLIVEIRA, K. C. V.; BARROS, A. L. S.; SOUZA, G. F. M. Mini-Exame do Estado Mental (MEEM) e Clinical Dementia Rating (CDR) em idosos com Doença de Alzheimer. Rev Neurocienc., São Paulo, v. 16, n. 2, p. 101-106, 2008.

SANTOS, C. S. et al. Avaliação da confiabilidade do mini-exame do estado mental em idosos e associação com variáveis sociodemográficas. Cogitare enferm., Curitiba, v. 15, n. 3, p. 406-412, jul./dez. 2010.

SANTOS, P. L.; FORONI, P. M.; CHAVES, M. C. F. Atividades físicas e de lazer e seu impacto sobre a cognição no envelhecimento. Medicina, Ribeirão Preto, v. 42, n. 1, p. 5460, 2009.

SANVITO, W. L. Propedêutica neurológica básica. 2. ed. Rio de Janeiro: Atheneu, 2010. $162 \mathrm{p}$.

SILVA, E. M. M. et al. Enfermidades do Paciente Idoso. Pesqui Bras Odontopediatria Clin Integr., João Pessoa (PB), v. 7, n. 1, p. 83-88, jan./abr. 2007. 